PROCEEDINGS OF THE

AMERICAN MATHEMATICAL SOCIETY

Volume 111, Number 4, April 1991

\title{
HOLOMORPHIC FUNCTIONS OPERATING IN HERMITIAN BANACH ALGEBRAS
}

\author{
A. ELKINANI
}

(Communicated by John B. Conway)

\begin{abstract}
The purpose of this paper is to prove that Hermitian algebras are the natural framework for the results of Ky Fan on analytic functions of a proper contraction.
\end{abstract}

\section{INTRODUCTION}

Let $A$ be an algebra over the complex field with the involution $x \rightarrow x^{*}$ and norm \|\| . The real and imaginary parts of an element $x$ of $A$ are denoted by $\operatorname{Re} x$ and $\operatorname{Im} x$, respectively; i.e., $\operatorname{Re} x=\left(x+x^{*}\right) / 2, \operatorname{Im} x=\left(x-x^{*}\right) / 2 i$. The spectrum and spectral radius of an element $x$ of $A$ will be denoted by $\operatorname{Sp} x$ and $\rho(x)$, respectively. The set of all Hermitian elements (i.e., all $x$ such that $x^{*}=x$ ) will be denoted by $H$.

We say that the algebra $A$ is Hermitian if the spectrum of every element of $H$ is real [9]. For elements $h$ and $k$ of $H$, we write $h \geq k$ to indicate that $h-k$ is positive, i.e., that $\operatorname{Sp}(h-k) \subset[0,+\infty[$. The notation $h>k$ will mean that $h-k$ is positive and invertible. Let $x$ be an element of $A$. We denote by $|x|$ the square root of the spectral radius of the element $x^{*} x$, i.e., $|x|=\rho\left(x^{*} x\right)^{1 / 2}$. In [9], V. Ptàk proved the following result: If $A$ is Hermitian, then the function $|\cdot|$ is an algebra seminorm on $A$ such that $\rho(x) \leq|x|$ for all $x \in A$.

We will use the following result of Shirali-Ford [10]:

$$
A \text { Hermitian } \Rightarrow x^{*} x \geq 0 \text {, for all } x \in A \text {. }
$$

Let $U$ be an open subset of the complex plane. We denote by $h(U)$ (resp., $H(U))$ the set of all harmonic (resp., holomorphic) functions on $U$.

Throughout the paper, $A$ will denote a complex unital Hermitian Banach algebra with continuous involution, and $D$ will denote the open disk $D=$ $\{Z:|Z|<1\}$ in $\mathbb{C}$. Also, $e$ will denote the unit (not necessarily of norm one) of $A$, and for scalars $r$ we often write simply $r$ for the element $r e$ of $A$.

Received by the editors December 31, 1988 and, in revised forms, May 1, 1989, November 13, 1989, January 26, 1990, and May 17, 1990.

1980 Mathematics Subject Classification (1985 Revision). Primary 46K99; Secondary 46H30.

Key words and phrases. Hermitian algebra, harmonic functional calculus, Hermitian element, invertible element. 


\section{Definition AND Lemmas}

In [1], we defined and studied the harmonic functional calculus. We recall the following definition:

Definition 2.1 [1]. Let $A$ be a complex unital Banach algebra with continuous involution, $U$ an open subset of $\mathbb{C}, Z_{0} \in U$ such that $\overline{D\left(Z_{0}, R\right)} \subset U(R>0)$, $x$ an element of $A$ with $\operatorname{Sp} x \subset D\left(Z_{0}, R\right)$ and $f \in h(U)$. Then

$$
f(x)=\frac{1}{2 \pi} \int_{\left|Z-Z_{0}\right|=R} f(Z) \operatorname{Re}\left[\left(Z+x-2 Z_{0}\right)(Z-x)^{-1}\right] \frac{|d Z|}{R} .
$$

We shall be concerned with the following classes of functions:

$$
\begin{aligned}
& B(D)=\{f \in H(D):|f(Z)|<1 \text { for } Z \in D\}, \\
& P(D)=\{g \in H(D): \operatorname{Re} g(Z)>0 \text { for } Z \in D\} .
\end{aligned}
$$

Lemma 2.2 (from [2, Theorem 33.1]). If $h \in A, k \in A, h \geq 0$, and $k \geq 0$, then $h+k \geq 0$.

Lemma 2.3. Let $x$ be an element of $A$. Then $|x|<1 \Leftrightarrow e-x^{*} x>0$.

Proof. First, assume $|x|<1$. It is clear that $e-x^{*} x$ is Hermitian, and it is invertible in $A$, since $\rho\left(x^{*} x\right)=|x|^{2}<1$. By the spectral mapping theorem, we have

$$
\operatorname{Sp}\left(e-x^{*} x\right)=\left\{1-\lambda: \lambda \in \operatorname{Sp} x^{*} x\right\} .
$$

It follows from (1) that $\operatorname{Sp} x^{*} x \subset[0, r]$, where $r=|x|^{2}<1$. Thus $\beta>0$ for every $\beta \in \operatorname{Sp}\left(e-x^{*} x\right)$. We prove the converse. By (2), we obtain $1-\lambda>0$ for every $\lambda \in \operatorname{Sp} x^{*} x$. This implies that $\max _{\lambda \in \operatorname{Sp} x^{*} x^{\lambda}}<1$. Hence, by (1), $|x|<1$.

Remark. If $A$ is a $\mathbb{C}^{*}$-algebra, it is clear that $|\cdot|$ can be replaced by $\|\cdot\|$. But this version is not true in general if $A$ is a Hermitian algebra, as the following example shows:

Let $A$ be the space of all complex-valued continuously differentiable mappings on $[0,1]$ with pointwise definition of addition, scalar multiplication, product, and the norm $\|f\|_{1}=\|f\|_{\infty}+\left\|f^{\prime}\right\|_{\infty}$, where $\|f\|_{\infty}=\operatorname{Sup}_{t \in[0,1]}|f(t)|$. It is easy to verify that $\left(A,\|\|_{1}\right)$ is a Hermitian algebra but not a $\mathbb{C}^{*}$-algebra. Now let $x$ be given by $x(t)=t^{2}-\frac{1}{2}$ for $t \in[0,1]$. It is clear that $e-x^{*} x>0$, but $\|x\|_{1}>1$.

We return now to a general Hermitian algebra $A$.

Lemma 2.4. Let $x$ be a Hermitian element of $A$. Then

$$
x \geq 0 \Leftrightarrow|| x|-x| \leq|x| .
$$


Proof. The assertion is trivial for $x \in \operatorname{Radical}(A)$, since then $\operatorname{Sp} x=\{0\}$, so assume $x \notin \operatorname{Radical}(A)$. Then, by homogeneity, we can further suppose that $|x|=1$. First, suppose $x \geq 0$ and $|x|=1$. Then

$$
\operatorname{Sp}(e-x)=\{1-\lambda: \lambda \in \operatorname{Sp} x\} \subset[0,1] .
$$

It follows that $|e-x|<1$.

Suppose now that $x$ is a Hermitian element of $A$, and since $|e-x|<1$, we have $1-\lambda \leq 1$ for every $\lambda \in \operatorname{Sp} x$. Whence $\lambda \geq 0$, for every $\lambda \in \operatorname{Sp} x$; and hence, $x \geq 0$.

Remark. By Lemma 2.4, the positive cone is $|\cdot|$-closed, and since $|\cdot| \leq \alpha\|\cdot\|$ for some $\infty>\alpha>0$, due to continuity of the involution, it is norm-closed.

\section{SOME APPLICATIONS OF THE HARMONIC CALCULUS}

3.1. A theorem of Ky Fan. Now we are ready to extend Fan's theorem (i.e., [3, Theorem 1]) to Hermitian algebras.

Theorem 3.1. Let $x \in A$, with $|x|<1$. Then $|f(x)|<1$ for every $f \in B(D)$, and $\operatorname{Re} g(x)>0$ for every $g \in P(D)$.

Proof. Let $f, g, x$ satisfy the hypothesis of Theorem 3.1. Since $\rho(x) \leq|x|$, it follows that $\operatorname{Sp} x \subset D$. Thus $f(x)$ and $\operatorname{Re} g(x)$ are defined.

By using Fan's proof [3, Theorem 1] and our Lemma 2.3, the reader can prove that the two assertions in this theorem are equivalent.

Now we prove the second assertion. Let $g \in P(D)$. Then $\operatorname{Re} g \in h(D)$. Choose positive numbers $r$ and $r^{\prime}$ with $|x|<r<r^{\prime}<1$. Then it is easy to verify that there exists $\delta>0$ such that $\operatorname{Re} g>\delta$ on $\overline{D\left(0, r^{\prime}\right)}$. Consider $h$ defined by $h(z)=\operatorname{Re} g(z)-\delta$ for each $z$ in $\overline{D\left(0, r^{\prime}\right)}$. Since $\rho(x) \leq|x|<r<$ $r^{\prime}$, it follows that $\operatorname{Sp} x \subset D(0, r)$. Thus, by Definition 2.1, we have

$$
h(x)=\frac{1}{2 \pi} \int_{|z|=r} h(z) \operatorname{Re}\left[(z+x)(z-x)^{-1}\right] \frac{|d z|}{r} .
$$

For all $z$ with $|z|=r$, we have $\operatorname{Re}\left[(z+x)(z-x)^{-1}\right] \in H$. Furthermore,

$$
\begin{aligned}
& \operatorname{Re}\left[(z+x)(z-x)^{-1}\right]=\frac{1}{2}\left[(z+x)(z-x)^{-1}+\left(\bar{z}-x^{*}\right)^{-1}\left(\bar{z}+x^{*}\right)\right] \\
& \quad=\frac{1}{2}\left(\bar{z}-x^{*}\right)^{-1}\left\{\left(\bar{z}-x^{*}\right)(z+x)+\left(\bar{z}+x^{*}\right)(z-x)\right\}(z-x)^{-1} \\
& \quad=\left(\bar{z}-x^{*}\right)^{-1}\left(r^{2}-x^{*} x\right)(z-x)^{-1} .
\end{aligned}
$$

It follows from Lemma 2.3 and the choice of $r$ that $r^{2}-x^{*} x>0$, so that $r^{2}-x^{*} x=u^{2}$ for some $u \in H$. Hence

$$
\operatorname{Re}\left[(z+x)(z-x)^{-1}\right]=\left(\bar{z}-x^{*}\right)^{-1} u \cdot u(z-x)^{-1} .
$$

Then, by (1), we have

$$
\operatorname{Re}\left[(z+x)(z-x)^{-1}\right] \geq 0 .
$$


Let us return to (3); the integral on the right-hand side is a limit of sums like

$$
h_{n}(x)=\sum_{k=0}^{n-1} \frac{1}{2 \pi} h\left(r e^{i \theta_{k}}\right) \operatorname{Re}\left[\left(r e^{i \theta_{k}}+x\right)\left(r e^{i \theta_{k}}-x\right)^{-1}\right]\left(\theta_{k+1}-\theta_{k}\right),
$$

where $\theta_{k} \in[0,2 \pi]$ for all $k \in\{0,1,2, \ldots, n\}$ and $0=\theta_{0}<\theta_{1}<\cdots<\theta_{n}=$ $2 \pi$. It is clear, by Lemma 2.2 , that $h_{n}(x) \geq 0$ and, since the positive cone is norm-closed, by the remark after Lemma $2.4, \lim _{n \rightarrow+\infty} h_{n}(x) \geq 0$. So we have $h(x) \geq 0$. Finally, since $h(x)=\operatorname{Re} g(x)-\delta$, we have $\operatorname{Sp} \operatorname{Re} g(x) \subset[\delta,+\infty[$. This completes the proof.

3.2. A theorem of von Neumann. In [3], Ky Fan proves that his result [3, Theorem 1] is closely related to the important theorem of von Neumann [3, Theorem 2]. We prove here that Theorem 3.1 is equivalent to the following result, which generalizes von Neumann's theorem:

Theorem 3.2.1. Let $x \in A$, with $|x| \leq 1$, and let $f$ be holomorphic on some neighborhood of the closed unit disk $\bar{D}=\{z:|z| \leq 1\}$. If $|f(z)| \leq 1$ for $z \in \bar{D}$, then $|f(x)| \leq 1$.

Proof. Theorem 3.2.1 is derived from Theorem 3.1 by using K. Fan's proof in [3] and the continuity of the holomorphic calculus.

Conversely, Theorem 3.1 follows from Theorem 3.2.1. In fact, a direct consequence of Theorem 3.2.1 is the following result, which clearly implies Theorem 3.1 .

Theorem 3.2.2. Suppose $f \in H(D)$. For $0<r<1$, let

$$
M(r)=\max \{|f(z)|:|z|=r\} .
$$

Then for such $r$

$$
M(r)=\max \{|f(x)|:|x| \leq r\},
$$

where the maximum is taken over all elements $x$ of $A$ such that $|x| \leq r$.

Proof. K. Fan's proof of [3, Theorem 3] applies to this case as well.

3.3. Analog of Schwarz's lemma. The analog of Schwarz's lemma is the following:

Theorem 3.3.1. Let $x \in A$, with $|x|<1$. Let $f, g, h \in H(D)$ be such that $f=g, h$ and $|h(z)| \leq 1$ for $z \in D$. Then

$$
g(x)^{*} g(x) \geq f(x)^{*} f(x)
$$

and

$$
|g(x)| \geq|f(x)| \text {. }
$$

Strict inequality holds in (5) if and only if $g(x)^{*} g(x)>0$ and $h$ is not a unimodular constant. Equality holds in (6) if and only if either $g(x) \in \operatorname{Radical}(A)$ or $h$ is a unimodular constant.

Proof. If $h$ is a constant function of absolute value 1 , it is clear that equality holds in both (5) and (6). 
On the other hand, if $h$ is not a constant function of absolute value 1, then $h \in B(D)$, by the maximum modulus principle. Thus $|h(x)|<1$, by Theorem 3.1. Consider a fixed $r$ such that $|h(x)|<r<1$. Since $f(x)=h(x) g(x)$, we have

$$
\begin{aligned}
r^{2} g(x)^{*} g(x)-f(x)^{*} f(x) & =r^{2} g(x)^{*} g(x)-g(x)^{*} h(x)^{*} h(x) g(x) \\
& =g(x)^{*}\left[\left(r^{2}-h(x)^{*} h(x)\right] g(x) .\right.
\end{aligned}
$$

Since $|h(x)|<r, r^{2}>h(x)^{*} h(x)$, and so it follows from the preceding equality and (1) that

$$
r^{2} g(x)^{*} g(x)-f(x)^{*} f(x) \geq 0 .
$$

Hence

$$
-f(x)^{*} f(x) \geq-r^{2} g(x)^{*} g(x)
$$

so that

$$
g(x)^{*} g(x)-f(x)^{*} f(x) \geq\left(1-r^{2}\right) g(x)^{*} g(x) .
$$

Then from (7) we deduce

$$
g(x)^{*} g(x) \geq f(x)^{*} f(x),
$$

with strict inequality in case $g(x)^{*} g(x)>0$, and only in this case. Now $|f(x)|=|h(x) g(x)|<r|g(x)|$, since $|h(x)|<r$, so we have $|f(x)|<|g(x)|$ in case $|g(x)| \neq 0$, i.e., in case $g(x) \notin \operatorname{Radical}(A)$. This completes the proof.

Remark. Clearly, Theorem 3.1 is a special case (i.e., $g=1$ ) of Theorem 3.3.1. Another special case of Theorem 3.3.1 is the following version of Jensen's inequality.

Corollary 3.3.2. Let $x \in A$, with $|x|<1$. Let $f \in B(D)$, and let $z_{1}, z_{2}, \ldots$, $z_{n}$ be some (not necessarily all) of the zeros of $f$ in $D$, each of which is allowed to repeat as often as its multiplicity. Let

$$
g(z)=\prod_{k=1}^{n} \frac{z-z_{k}}{1-\bar{z}_{k} z} .
$$

Then we have (5) and (6). Strict inequality in (5) holds if and only if $g(x)^{*} g(x)>$ 0 and $f$ is not of the form $\eta g$ for some constant $\eta$ with $|\eta|=1$. There is equality in (6) if and only if either $g(x) \in \operatorname{Radical}(A)$ or $f=\eta g$ for some unimodular constant $\eta$.

Proof. K. Fan's proof of [3, Corollary 1] applies, mutatis mutandis, to this case as well.

In the case $z_{1}, z_{2}=\cdots=z_{n}=0$, Corollary 3.3.2 becomes the following 
form of Schwarz's lemma:

Corollary 3.3.3. Let $x \in A$, with $|x|<1$. Let $f \in B(D)$ be such that $f(0)=$ $f^{\prime}(0)=\cdots=f^{(n-1)}(0)=0$ for some integer $n \geq 1$. Then

$$
x^{n *} x^{n} \geq f(x)^{*} f(x)
$$

and

$$
\left|x^{n}\right| \geq|f(x)| \text {. }
$$

Strict inequality holds in (8) if and only if $f$ is not of the form $f(z)=\eta z^{n}$ with unimodular constant $\eta$ and $x^{n *} x^{n}>0$, and equality holds in (9) if and only if either $x^{n} \in \operatorname{Radical}(A)$ or $f(z) \equiv \eta z^{n}$ with unimodular constant $\eta$.

Remark. If $h \in A, k \in A, 0 \leq h \leq k$, then $|h| \leq|k|$. In fact, it is clear that $|k|-k \geq 0$, since $|k|=\rho(k)$. By Lemma 2.2 , we have $|k|-k+k-h \geq 0$, i.e., that $|k|-h \geq 0$. This implies that $|h| \leq|k|$.

An immediate consequence of Corollary 3.3.3 is the following extension of Harnack's double inequality:

Corollary 3.3.4. Let $x \in A$, with $|x|<1$. Let $n$ be a positive integer and $g \in P(D)$ be such that $g(0)=1$. In the case $n \geq 2$, suppose also that $g^{\prime}(0)=$ $g^{\prime \prime}(0)=\cdots=g^{(n-1)}(0)=0$. Then

$$
\left[e+g(x)^{*}\right] x^{n *} x^{n}[e+g(x)] \geq\left[e-g(x)^{*}\right][e-g(x)],
$$

and

$$
\frac{1-\left|x^{n}\right|}{1+\left|x^{n}\right|} \leq|g(x)| \leq \frac{1+\left|x^{n}\right|}{1-\left|x^{n}\right|} .
$$

Proof. It is obtained as in [3], by using Corollary 3.3.3, (1), and the preceding remark.

A special case of Corollary 3.3.4 is the following analog of Caratheodory's inequality.

Corollary 3.3.5. Let $x \in A$, with $|x|<1$. If $h \in H(D), h(0)=h^{\prime}(0)=\cdots=$ $h^{(n-1)}(0)=0$ for some $n \geq 1$, and $\operatorname{Re} h(z)<1$ for $z \in D$, then

$$
|h(x)| \leq \frac{2\left|x^{n}\right|}{1-\left|x^{n}\right|}
$$

Proof. We use K. Fan's proof of [3, Corollary 4] and the preceding remark.

By using our Corollary 3.3.3, where K. Fan uses his Corollary 2 in the proofs of his [3, Theorems 5-7], the holomorphic functional calculus, and (1), the reader can prove our Theorems 3.4.1, 3.5.1, and 3.6.1 below.

3.4. Analog of Pick's theorem. The generalization of the case $n=1$ of Corollary 3.3.3 is the following analog of Pick's theorem. 
Theorem 3.4.1. Let $x \in A$, with $|x|<1$. Let $f \in B(D)$ and $z_{0} \in D$. Then (12)

$$
\begin{aligned}
& \left(e-z_{0} x^{*}\right)^{-1}\left(x^{*}-\bar{z}_{0}\right)\left(x-z_{0}\right)\left(e-\bar{z}_{0} x\right)^{-1} \\
& \quad \geq\left\{e-f\left(z_{0}\right) f(x)^{*}\right\}^{-1}\left\{f(x)^{*}-\overline{f\left(z_{0}\right)}\right\}\left\{f(x)-f\left(z_{0}\right)\right\}\left\{e-\overline{f\left(z_{0}\right)} f(x)\right\}^{-1},
\end{aligned}
$$

and

$$
\left|\left(x-z_{0}\right)\left(e-\bar{z}_{0} x\right)^{-1}\right| \geq\left|\left\{f(x)-f\left(z_{0}\right)\right\}\left\{e-\overline{f\left(z_{0}\right)} f(x)\right\}^{-1}\right| .
$$

Strict inequality in (12) holds if and only if $\left(x^{*}-\bar{z}_{0}\right)\left(x-z_{0}\right)>0$ and is not of the form

$$
f(z)=\varepsilon \frac{z-z_{1}}{1-\bar{z}_{1} z} \quad \text { with }|\varepsilon|=1,\left|z_{1}\right|<1 .
$$

Equality holds in (13) if and only if either $x=z_{0}$ or $f$ is of the form (14).

3.5. Vitali's convergence theorem. We obtain the following extension of Vitali's convergence theorem:

Theorem 3.5.1. Let $\left(x_{k}\right)_{k}$ be a sequence of invertible elements of $A$ such that $\left|x_{k}\right|<1$ for all $k$ and $\lim _{k \rightarrow \infty}\left|x_{k}\right|=0$. Let $\left(f_{n}\right)_{n}$ be a sequence of functions in $H(D)$ that is uniformly bounded on $D$. If for each $k,\left\{f_{n}\left(x_{k}\right)\right\}_{n=1}^{\infty}$ is a $|\cdot|$-Cauchy sequence, then there is an $f \in H(D)$ such that $\lim _{n \rightarrow \infty} f_{n}(z)=f(z)$ uniformly on every compact subset of $D$.

An immediate consequence of this result is the following identity theorem:

Corollary 3.5.2 (Identity theorem). Let $f, g \in H(D)$. If there is a sequence $\left(x_{k}\right)_{k}$ of invertible elements of $A$ such that $\left|x_{k}\right|<1$ for all $k, \lim _{k \rightarrow+\infty}\left|x_{k}\right|=0$ and $f\left(x_{k}\right)=g\left(x_{k}\right)$ for each $k$, then $f=g$.

Remark. [3, Example 3] shows that the invertibility of the $\left(x_{k}\right)_{k}$ is necessary in Corollary 3.5.2.

3.6. Principle of subordination. A direct consequence of Corollary 3.3.3 and the classical principle of subordination is the following result, which has a number of interesting applications:

Theorem 3.6.1. Let $g \in H(D)$ be univalent on $D$ with $g(0)=0$. If $f(0)=0$ and $f(D) \subset g(D)$, then for every element $x$ of $A$ such that $|x|<1$, there is a unique element $y$ on $A$ such that $|y|<1$ that satisfies

$$
f(x)=g(y) \text {. }
$$

Moreover,

(i) $x^{*} x \geq y^{*} y,|x| \geq|y|$.

(ii) $x^{*} x>y^{*} y$ if and only if $x^{*} x>0$ and $f$ is not of the form $f(z) \equiv g(\eta z)$ for some constant $\eta$ with $|\eta|=1$.

(iii) $|x|=|y|$ if and only if either $x \in \operatorname{Radical}(A)$ or $f$ is of the form $f(z) \equiv g(\eta z)$ with unimodular constant $\eta$. 
A simple application of this result is the following extension of KoebeBieberbach's $\frac{1}{4}$-theorem for univalent functions.

Corollary 3.6.2. Let $g \in H(D)$ be univalent on $D$, and $g(0)=0, g^{\prime}(0)=1$. For every element $x$ of $A$ with $|x|<\frac{1}{4}$, there is a unique element $y$ in $A$ such that $|y|<1$ and $g(y)=x$. Moreover, $16 x^{*} x \geq y^{*} y$, with strict inequality in the case where $x^{*} x>0$, and $|y|<4|x|$ unless $x \in \operatorname{Radical}(A)$.

Proof. K. Fan's proof of [3, Corollary 6] applies here, too. In case $g(D)$ is a convex set, the constants $\frac{1}{4}, 16,4$ in Corollary 3.6 .2 can be improved to $\frac{1}{2}, 4,2$, respectively.

Corollary 3.6.3. Let $g \in H(D)$ be univalent on $D$ with $g(0)=0$. If $g$ is starlike (i.e., the image $g(D)$ is a starlike set with respect to the origin), then the set of all $g(x)$, where $x$ runs through all elements of $A$ such that $|x|<1$, is a starlike subset of $A$. More precisely: for every element $x$ of $A$ with $|x|<1$ and any nonnegative real number $r<1$, there is a unique element $y$ in $A$ with $|y|<1$ such that $g(y)=r g(x)$. Moreover, $x^{*} x \geq y^{*} y$, with strict inequality in case $x^{*} x>0$, and $|x|>|y|$ unless $x \in \operatorname{Radical}(A)$.

Proof. K. Fan's proof of [3, Corollary 7] applies to this case as well.

3.7. Convex univalent functions. Let $K(D)$ denote the class of univalent functions $f$ in $H(D)$ with $f(0)=0, f^{\prime}(0)=1$ and such that the image $f(D)$ is a convex set.

By using K. Fan's proof of his [3, Theorem 8] together with our Lemma 2.3 and (1), the reader can prove the following result:

Theorem 3.7. Let $\eta$ be a complex number with $|\eta|=1$, and let $h_{\eta} \in K(D)$ be the functions $h_{\eta}(z):=z(1-\eta z)^{-1}$. When $x$ runs through all elements in $A$ with $|x|<1$ and $\eta$ is kept fixed, the set of all $h_{\eta}(x)$ is a convex subset of $A$.

In [3], Ky Fan leaves open the question whether this is valid for every $h \in$ $K(D)$, but a counterexample was produced by Hwang in [8].

3.8. Typically real functions. Let $T R(D)$ be the set of those functions $f$ in $H(D)$ that satisfies the condition $(\operatorname{Im} z) \cdot(\operatorname{Im} f(z)) \geq 0$ on $D$ and $f(0)=0$, $f^{\prime}(0)=1$.

Theorem 3.8. Let $x \in A$, with $|x|<1$. Then $\operatorname{Im} f(x) \geq 0$ for every $f \in T R(D)$ if and only if $\operatorname{Im} x \geq x^{*}(\operatorname{Im} x) x$.

Proof. K. Fan's proof of [3, Theorem 9] applies to this case as well.

\section{FINAL REMARKS}

1. Von Neumann has proved (cf. [3]) the following spectral inequality:

$$
f \in H(D), \quad|f|<1, \quad T \in \mathscr{B}(\mathscr{H}), \quad\|T\|<1 \Rightarrow\|f(T)\|<1 .
$$

In [5], Foias showed that to replace $\mathscr{H}$ by a Banach space $E$ is not a genuine generalization, since $E$ must then be Hilbert after all. Here we have generalized 
the relation (15) to the case where $\mathscr{B}(\mathscr{H})$ is replaced by a unital Banach algebra with continuous Hermitian involution.

2. Fan's proof of [3, Theorem 1] uses the extreme points of the Montel space of contractive holomorphic functions on $D$. A simplified proof is given in [6], using a weak maximum principle in complex Banach algebras. In [11], a new Hilbert space norm equivalent to the original is introduced and used to prove the analog of Ky Fan's theorem for commuting topological proper contractions. In this paper we have extended Fan's theorem to Hermitian algebras.

\section{ACKNOWLEDGMENT}

The author thanks the referee for his remarks and valuable suggestions.

I have had many fruitful conversations with Prof. M. Oudadess. It is a great pleasure for me to express my sincere gratitude to him for his help and his advice.

\section{REFERENCES}

1. M. Akkar, A. Elkinani, and M. Oudadess, Calculus fonctionnels harmonique et analytique réel, Ann. Sci. Math. Québec 12 (1988), 151-169.

2. R. S. Doran and V. A. Belfi, Characterizations of $\mathbb{C}^{*}$-algebras, Marcel Dekker, New York, 1986.

3. K. Fan, Analytic functions of a proper contraction, Math. Z. 160 (1978), 275-290.

4. __ Sharpened forms of an inequality of von Neumann, Math. Z. 194 (1987), 7-13.

5. C. Foias, Sur certains théorèmes de J. von Neumann concernant les ensembles spectraux, Acta Sci. Math. (Szeged) 18 (1957), 15-20.

6. Chen Gong-Ning, On simplified proofs of theorems of von Neumann, Heinz, and Ky Fan, and their extended versions, in Nonlinear and convex analysis (Bor-Luh Lin and Steve Simons, eds.), Lecture Notes in Pure and Appl. Math. 107, Marcel Dekker, New York, 1987, pp. $15-21$.

7. __ A note on Vitali's convergence theorem, Beijing Shifan Daxue Xuebao (1983), 17-22.

8. J. S. Hwang, A problem on the Riesz-Dunford operator calculus and convex univalent functions, Glasgow Math. J. 24 (1983), 129-130.

9. V. Pták, Banach algebras with involution, Manuscripta Math. 6 (1972), 245-290.

10. S. Shirali and J. W. M. Ford, Symmetry in complex involutory Banach algebras. II, Duke Math. J. 37 (1970), 275-280.

11. M. H. Shih and K.-K. Tan, Analytic functions of topological proper contractions, Math. Z. 187 (1984), 317-323.

Department of Mathematics, Ecole Normale Superieure de Takaddoum, Boite Postale: 5118 Rabat, Maroc 